\title{
SUN'IY INTELLEKNI RIVOJLANTIRISHDA TILNI QOLIPLASHTIRISHNING O`RNI
}

Abror Murtazayev

ToshDO'TAU mustaqil izlanuvchisi Email: abror.murtazayev.89@inbox.ru

Annotasiya. Ushbu maqolada ilmiy matnlardagi aynan o'xshashlik, uning aniqlash uchun tilni qoliplashtirish, sodda darak gaplar(misolida)ning o'zaro sinonimlik hodisasining o'rni haqida fikr yuritiladi.

Kalit so'zlar: gap, sun'iy intellekt, lingvistika, qoliplashtirish, ilmiy matn, sinonimiya, aynan o'xshashlik, darajalanish, mualliflik huquqi.

Annotation. This article discusses the exact similarity in scientific texts, the importance of its definition, the role of language in the formation, in particular, the method of mutual synonymy of simple sentences.

Key words: speech, artificial intelligence, linguistics, modeling, scientific text, synonymy, exact similarity, ranking, copyright.

Аннотации. В статье обсуждается точное сходство научных текстов, важность его определения, роль языка в образовании, в частности, метод взаимной синонимии простых предложений.

O'zbekistonda ilmiy-tadqiqotning rivojlanishi, original ilmiy matnlarning paydo bo'lishi, muayyan lingvistik nazorat (ko'chirmakash-liklarning oldini olish uchun) tartibini ishlab chiqishni taqazo qiladi. Buning uchun elektron shaklda mavjud ilmiy matnlarning mazmuniy o'xshashlik darajasini aniqlaydigan dasturiy ta'minot yaratilishi zarur. Mazkur holat barcha rivojlangan mamlakatlar qatorida O'zbekistonda ham huquqiy jihatdan tartibga solinishi yo'lga qo'yildi. O'zbekiston Respublikasi Prezidentining "Intellektual mulk sohasida davlat boshqaruvini takomillashtirish chora-tadbirlari to 'g 'risida” 2019-yil 8-fevraldagi PQ-4168-son va "O'zbekiston Respublikasi Adliya vazirligi huzuridagi Intellektual mulk agentligi faoliyatini tashkil etish chora-tadbirlari to 'g 'risida" 2019-yil 1-iyuldagi PQ-4380-son qarorlari hamda shu qarorlarga muvofiq O'zbekiston Respublikasi Vazirlar Mahkamasining 2019-yil 20- iyuldagi 609-son qarori [Lex.uz] bilan huquqiy jihatdan tartibga solinishi belgilab qo'yilgan. Mualliflik huquqida qarshi kurashiladigan masala bu - ko'chirmakashlik. O`zbek tilining izohli lug'atida 
ko'chirmakash, ko'chirmakashlik, ko'chirmachi, ko'chirmachilik - O'zganing ishini ko'chirib olib, o'ziniki qilib ko'rsatuvchi kishi, O'zganing ishini ko'chirib olish bilan shug'ullanish. Bu umumiy izoh bo'lib, yuridik atama sifatidagi ma'nosi quyidagicha izohlangan: Adabiy o'g'irlik. Plagiat - birovning asarini ko'chirib, o'ziniki qilib olish. Bunday o'g 'irlik muallif ijozatisiz, ilmiy, adabiy, musiqiy yoki badiiy asarni o'z nomidan to 'la yoki qisman bostirib chiqarishdan, kashfiyot yoki ixtirochilik takliflarini o'ziniki qilib olish... [O'zbek tilining izohli lug'ati. 2020:56]. bundan ko'rinib turibdiki, qonunchilik ko'chirmakashlikning maqomini uning qay hollarda jinoyat ekanligini belgilab qo'ygan, biroq ish bu bilan tugmaydi. Biror ishni ko"chirmakashlik ekanligini o'sha soha mutaxassislari masalan, san'at asarini ko'chirilganligini rassom yoki haykaltaroshlar, ilmiy-badiiy asarning ko"chirmakashlik ekanligini esa soha olimlari aniqlab beradi. Bu masalda muammolarning ko'payishi oliy ta'limda amalga oshirilayotgan ilmiy ishlarni internet tarmog'iga joylashtirib borish bilan bog'liq. Yuzaga kelgan bunday vaziyat bugungi kunga kelib, dunyoda o'zganing intellektual mulkidan noqonuniy foydalanishga qarshi kurashishni yangi bosqichga ko'tarishni talab qilmoqda. Ushbu kurashning avjiga chiqishi Internetning keng tarqalishi, undan arzonroq foydalanish, (masofadan turib olinadigan xizmatlar) xizmatlar sonining ko'payishi, elektron tijorat tizimlarining mavjudligi bilan bog'liq. Bu borada qilingan ishlarni muallifning original ishi yoki ilmiy odobga amal qilinmay amalga oshirilgan o'g'rilik ekanligini aniqlash uchun inson omilining o'zi yetarli bo'lmay qoldi. Zero, biror sohadagi tadqiqotlar haqidagi batafsil ma'lumotlarni (barcha ilmiy ishlarni) bir yoki bir nechta insonda jamlash imkonsiz ekanligini bilishning o'zi fikrimizni dalillaydi. Inson tabiati doimo mashaqqatni xohlamasligi tabiiy bo'lgani holda buning ham yechimini izlaydi. Jahon tajribasida ko'chirmakashiliklarni oldini olish uchun maxsus elektron antiplagiat dasturlar ishlab chiqilgan va amaliyotda qo"llanilmoqda. Mazkur dasturlar matnlardagi aynanlikni teshirib, uning umimiy hajmidan kelib chiqqan holda noqonuniy o'zlashtirishlar miqdori foizlar hisobida ko'rsatib beriladi. Biroq internetda bunga qarshi ko'chirmachilik (plagiat)ni chetlab o'tishga doir turli tijoriy takliflar ham paydo bo 'ldi. Dastlab antiplagiat dasturini matndagi alifbolarni, jumladan, rus alifbosini masalan "a", "o" va "s" harflarini lotinga yoki aksincha qilish bilan ko"chirmakashlikni kamaytirishga erishildi va bu 2016-yilgacha foyda berdi.

Antiplagiat dasturini aldash uchun matn orasiga turli ko'rinmas belgi yoki harflarni kiritib, uni oq rangga bo'yash kabi usullardan ham foydalanilgan bo'lib, bular ham 2020-yilga kelib dasturni mukammalashtirish natijasida foyda bermay 
qo 'ydi. [Antiplagiat.ru] Antiplagiatning dastlabki turi "aynan bir xil matnlarni tekshirgan" bo"lsa, keyingi bosqichi - bu "niqoblangan", ya'ni alifbo harflarni boshqa alifboga o'zgartirib matn tayyorlash, turli ko'rinmas belgilar kabilarni qo'shilgan matnlarni aniqlashga xizmat qildi. Shuningdek, Antiplagiatning "mazmuniy o'xshashlikni”, "ko"p tilli" (tarijima ishlarni aniqlovchi) hamda "g'oyaning o'g'irlanganligini" aniqlovchi turlari haqida turli nazariyalar mavjud. Bugungi kunda matnlar o'rtasida mazmuniy o'xshashlikni aniqlovchi antiplagiat dasturiga ehtiyoj har doimgidan ko'ra yuqori ekanligini ta'kidlash lozim. Fikrimizning dalili sifatida 2019-yilda (ingliz va rus tillari uchun) "Aq1li sinonimizatorlar" [Antiplagiat.ru] deb nomlangan dasturlar avlodi paydo bo'lganligini ta'kidlashning o'zi kifoya. Vaqt o'tishi bilan o'ylab topilgan turli hiyla-nayranglar ham barham topgach, endi yana-da murakkab nayrang - "Aqlli sinonimizatorlar" paydo bo "ldi. Bu esa antiplagiat dasturining takomillashuvida kompyuter muhandislari bilan filolog mutaxassislarning professional hamkorligini talab qiladi.

Tilning ijtimoiy hodisa ekanligini tasdiqlovchi dalillar nihoyatda ko'p. Vaqt o'tishi bilan sodir bo'layotgan yangiliklar yangi fan sohalarini ham yuzaga kelishiga turki bo'lmoqda. Xuddi shunday yangi sohalardan biri kognitiv tilshunoslikdir. Tilshunoslik kognitologiya fani shakllanishida poydevor bo'lib xizmat qilganligi sababli uch soha - komp'yuter lingvistikasi, kognitiv psixologiya hamda generativ grammatika qatoridan bejiz joy olmagan[Safarov.Sh. 2006:19].

Amerikalik psixolog H.Gardner kognitiv fanlar kesishuvida falsafa, psixologiya, tilshunoslik, sun'iy intellekt, nevrologiya hamda antroplogiya fanlarini sanagan. Inson borliq va undagi narsalar haqida falsafiy tafakkur qiladi hamda buni til orqali tartibga soladi va taqdim etadi. Mavjud fanlarning dastlab falsafiy yondashishga ko'ra tadqiq etilishini e'tiborga olgan holda dastlab biz uchun muhim bo'Igan aynanlik tushunchasining falsafa va boshqa fanlardagi o'rni, mazmunmohiyatini yoritishdan boshladik.

Falsafada "Bilish nazariyasi"ga ko'ra, aynanlik bu aynan o'xshashlik (lotin./iden) so'zidan olingan bo'lib, o'shaning o'zi, aynan ma'nolarini ifodalaydi [Abdullaeva M va boshq. 2004:14]. Ya'ni aynanlik biror narsa yoki voqeahodisaning boshqasiga aynan o'xshash ekanligi, o'zaro bir-biriga to 'la muvofiqlik. Shu bilan birga mazkur fanda narsa va hodisalardagi o'zaro o'xshash tomonlar birligiga ayniyat deyiladi. Tafakkurda mavjud bo'lgan aynanlik tamoyili turli vaziyat va sharoitlarda $0^{6} \mathrm{z}-\mathrm{o}^{6} \mathrm{zining}$ aynanligini saqlab qolgan holdagina $\mathrm{o}^{6} \mathrm{z}-\mathrm{o}^{\text {' } z i g a}$ teng bo'ladi. Ayniyat tamoyili faqat fikrlash jarayonida, mushohada paytida 
qo'llaniladigan tushunchaning aynan bir ma'noda ishlatilishini talab qiladi. Narsa o'z-o'zigagina nisbatan aynan bo'lishi mumkin. Aynan narsa va hodisalardagi o'xshashlik hamda mutlaq tenglik (barcha muhim xususiyatlarning o'zaro birbiriga to' la mosligi) shakllarida namoyon bo'ladi.

Narsa-hodisalar ma'lum nisbatdagina o'z-o'ziga aynan bo'ladi, aslida $u$ doimo o'zgarishda bo'lgani uchun uning to'la, batamom aynanligi haqida fikr yuritish aksariyat hollarda noto'g'ri bo'ladi. Ong ham o'z-o'ziga aynan (teng) bo'lishi mumkin emas, u taraqqiy etib boradi, lekin bu taraqqiyot «men»ning taraqqiyoti tarzida kichadi [Abdullaeva M va boshq. 2004:14]. Bu o'rinda "nisbiylik nazariyasi”ni yodga olish zarur. Mazkur izohlardan falsafada aynanlik mavjud bo' lish bilan birga, narsa va hodisalarning ma'lum bir vaqtda aynan bir xil bo'lishi mumkin ekanligi hamda vaqt o'tishi va boshqa omillargan ko'ra voqeahodisalar hamda narsalarning o'zaro aynanlik holati yo'qolishi ham tabiiy hodisa ekanligi oydinlashadi. Borliq narsa, voqea-hodisalarning turli-tumanligidan iborat. Lekin narsalar qanchalik xilma-xil, turli-tuman bo'lmasin, ular o'rtasida yaqinlik, aynanlik mavjuddir [Nazarov Q. Falsafa.2000:173]. Bu aynanlik unga katta hajmdagi ma'lumotlarni o'xshatish va farqlash belgilari orqali tanib olish, foydalanish kabi o'rinlar uchun muhim ko'rsatkichdir. Tilshunoslikda ham aynanlik tushunchasi mavjud bo'lsa-da bu hodisa nazariy jihatdan jiddiy tadqiq etilmagan. "O'zbek tilining izohli lug'ati"da "Aynan [a. - xuddi o 'zi] rvsh. kt. 1 Qanday bo 'lsa, shunday, hech qanday o 'zgarishsiz. Aynan tarjima. Elmurod javob berish uchun ko 'p o 'ylab turmadi. Ilgari bo 'lgan voqeani bugunga aynan ko 'chirib aytib qo'ya qoldi. 2 yukl. Xuddi. Otasining aynan o'zi. .." [Nazarov Q. Falsafa.2000:173] izohidan ma'lumki, aynanlik hech istisnosiz bir xillikni, farqlar bo'lishini rad etadi. Lekin bu izohlarda keltirilgan misollarni tahlil qiladigan bo'Isak aynanlik mutlaq bo'lmasligi ham mumkin ekanligi ma'lum bo'ladi. Masalan, birinchi misolda voqea qanday bo'lsa aynan shunday aytib berish mumkin. Lekin, Otasining aynan o'zi deganda uning tashqi ko'rinishi yoki xarakter xususiyatlari bir ekanligini nazarda tutish mumkin. Ya'ni otasining aynan o'zi degan o'sha odamning to'liq o'rninini bosa oladi degan xulosani beramaydi. Bundan tashqari izohli lug'atda baravar, teng, bir, birday, bir xil, dublet (fr) 1 Biror narsaning ikkinchi nusxasi; ikkita bir xil, teng qimmatli narsaning biri., dublyor (fr) 1 Biror ishni navbatlashib bajaruvchi yoki bir ishda bir-birining o 'rnini bosa oluvchi ikki kishidan biri., dublikat (fr) Biror yozma hujjatning asl nusxasi bilan bab-baravar rasmiy, yuridik kuchga ega bo 'lgan boshqa nusxasi, [O'zbek tilining izohli lug'ati. 2020:56] kabi so'zlar bo'lib, aynanlik tushunchasini turli tomondan 
turli o'rinlarda ifodalash uchun qo'llaniladi.

Biz tilshunoslikda aynanlikni sodda gaplar va ular tarkibidagi so'zlar miqyosida o'raganamiz. Albatta, so'z va gap filologiyada eng muhim tushunchalardan biri. Har bir so' $z$ muayyan tushuncha yoki fikrni ifodalashga xizmat qiladi. Tabiiyki, so'z yoki so'zlar birikmasi grammatik jihatdan shakllanib, gaplarni hosil qiladi. Hosil bo'lgan gaplar muayyan fikrni ifodalashga xizmat qiladi. Gapning mazmuniga qiziqish undagi turli so'zlarga xos turfa ma'nolarining ochilishiga olib keladi. So 'zlarning shakl va ma'nosiga doir tadqiqotlar natijasida sinonimlik, antonimlik va giponimlik hodisalari ma'lum bo'ldi. Biz mavzu doirasida mazkur tushunchalar haqida ham to 'xtalamiz. Tilshunoslikda sinonimiya - shaklan har xil, ammo bir tushunchani turli bo'yoq va ottenka tusi bilan ifodalaydigan leksemalar sinonim deyiladi. (gr. synonymos - "bir nomli"). Sinonim leksema orasidagi munosabat sinonimiya yoki sinonimik munosabat deb yuritiladi. [Sayfullayeva R., Mengliyev B.va boshq. 2010:152]

Mazkur qoidaga binoan sinonim deb qaralayotgan leksemalarning semalaridagi atash hamda vazifa semasi aynan bo'lgani holda ifoda semasida farqlar mavjud ekanligi ta'kidlanadi. Misol sifatida yuz - bet - aft - bashara - turq so'zlarining barchasi tepadan odam peshanosining soch bitgan joyidan toki iyaklari ostigacha, ikki yon tarafdan ikki quloqlarining yumshoqlarigacha bo'lgan chegara qismni ifodalovchi so "zni tushunish mumkin. Biroq ifoda semalarida muayyan tafovutlar bor, ya'ni, "shaxsiy munosabat": yuz leksemasida "shaxsiy betaraf munosabat", turq leksemasida "o'ta kuchli shaxsiy munosabat" holatida bo'ladi. $\mathrm{Bu}$ o'rinda keltirilgan misolda namoyon bo'lgan aynanlik mutlaq emasligi ma'lum bo'ladi. Sinonimlarga xos xususiyat so'z ma'nosida aynanlikka xos, ya'ni biri o'rnida ikkinchisini qo'llashning ustun ekanligi bilan xarakterlanadi. Sinonimiya hodisasi so'zlar bilan iboralar, ibora va ibora o'rtasida ham uchraydi va bu borada ko'plab ilmiy izlanishlar amalga oshirilgan. Endigi vazifa esa, gaplarda sinonimiya hodisasini tadqiq etish. Masalan, Men maktabda ishlayman - Men maktabda faoliyat yuritaman - Men maktab o'qituvchisiman. Ushbu gaplar umumiy holda sinonim bo'la oladi. Birinchi va ikkinchi gaplarda vazifasining aniq emasligi nuqtai nazaridan o'zaro sinonimlik yuqoriroq bo'lsa-da, uchinchi gapni avvalgi har ikkala gap bilan ifodalashga ko'ra sinonimlik hosil qiladi deyish mumkin.

Aynanlik kategoriya sifatida tilagi mavjud tushunchalar ularning aloqadorliklari va o'zaro ta'sir doiralarini aniqlashtirib berishda hamda ulardan foydalanish samaradorligini oshirishda alohida ahamiyatga ega. Zero, tildagi darajalanish umumiy borliqning o'zida mavjud bo'lgan darajalanish bilan 
shartlangandir. [ Bozorov O. 1997:25] Ya'ni darajalanish aynanlik uchun ham o'rinli hodisa sifatida mutlaq yoki nisbiy bo'lishi mumkin.

Aynanlik va o'xshashlik, farqlilik falsafiy, ijtimoiy nuqtai nazardan ijobiy hodisa sifatida qaralishi mumkin. Ammo ilmiy jihatdan matnlarni, tafakkur mahsuli hisoblangan o'ziga xos (original) fikrlarni o'xshash bo'lishi to ' $\mathrm{g}$ 'rirog' $\mathrm{i}$ aynan o'xshash bo'lishi ijobiy hodisa emas. Shu nuqtayi nazardan matn yaratishda o'zgalar fikridan foydalanish bir qator axloqiy, ijtimoiy, huquqiy muammolarni keltirib chiqaradi. Shu ma'noda globallashuv sharoitida tayyor ma'lumotdan foydalanish etikasi buzilgan holatlarda, ya'ni intellektual mulk hisoblangan fikr va g'oyalarni o'zlashtirilishi lingvistik ekspertiza hamda komp'yuter lingvistikasi sohalarini yanada rivojlantirishga turtki beradi. Mazkur ijtimoiy zarurat ilmiy matnlarda aynanlik, o'xshashlik va farqlilik hodisalarini maxsus, keng miqyosda monografik o'rganishni taqazo qiladi.

Shu o'rinda biz mavjud aynanlik, o'xshashlik tushunchalarini birlashtirish tarafdorimiz, ya'ni o'xshashlik ilmiy matnlarni tekshirish jarayonida mutlaq ish bermaydi. Zero, o'xshashlik nisbiy bo'lishi mumkin. Shu ma'noda biz "aynan o 'xshashlik" birikmasini taklif qilamiz. Tilda qoliplashtirish masalasi to "g'ri va aniq ishlab chiqilganda nazariy va amaliy jihatdan e'tibordan chetda qolgan hodisalarni, tilning keng va foydali imkoniyatlarini ochilishiga erishish mumkin.

\section{Foydalanilgan adabiyotlar:}

1. O'zbek tilining izohli lug'ati. V jildlik. 1-jild. - Toshkent: O'zbekiston milliy ensiklopediyasi, 2020. $56 \mathrm{~b}$

2. Safarov.Sh. Kognitiv tilshunoslik. Samarqand. 2006-yil

3. Abdullaeva M va boshq.Qisqacha falsafa lug'ati. Toshkent. 2004-yil

4. Nazarov Q. Falsafa .Toshkent. 2000-yil

5. Sayfullaeva R., Mengliyev B. va boshq. Hozirgi o'zbek adabiy tili Toshkent. 2010-yil.

6. Bozorov O. O`zbek tilida darajalanish. F.f. dok uchun diss.. Toshkent 1997-yil

7. Lex.uz

8. Ziyouz.com

9. Antiplagiat.ru 